\title{
Total aortic arch replacement with a novel 4-branched frozen elephant trunk prosthesis: Single-center results of the first 100 patients
}

\author{
Malakh Shrestha, MBBS, Tim Kaufeld, MD, Erik Beckmann, MD, Felix Fleissner, MD, \\ Julia Umminger, MD, Firas Abd Alhadi, MD, Dietmar Boethig, MD, Heike Krueger, RN, \\ Axel Haverich, MD, and Andreas Martens, MD
}

\begin{abstract}
Objective: Combined disease of the aortic arch and the proximal descending aorta remains a surgical challenge. The Thoraflex Hybrid graft (Vascutek, Inchinnan, United Kingdom) consists of a 4-branched graft with a stent graft at the distal end allowing a total aortic arch replacement, including the origins of the supra-aortic vessels combined with endoluminal treatment of the proximal descending aorta. We present the midterm results of our first 100 patients who were treated with this frozen elephant trunk prosthesis.
\end{abstract}

Methods: From April 2010 to October 2014, 100 patients (65 men aged $59 \pm 14$ years) underwent operation (37 acute dissections, 31 chronic dissections, and 32 aneurysms). Fifty-four percent of patients received concomitant cardiac procedures, and $28 \%$ were reoperations.

Results: The perioperative mortality was 7\% $(n=7)$. Midterm survival after a follow-up of $3.1 \pm 1.4$ years was $81 \%(\mathrm{n}=81)$. Mean cardiopulmonary bypass time was $243 \pm 61$ minutes, cardiac ischemia time was $101 \pm 65$ minutes, and circulatory arrest time was $51 \pm 20$ minutes. Aortic root replacement was performed in 41 patients $(n=41$; valve-sparing: $30 \%[n=30])$. Twenty-two percent of patients underwent secondary aortic reinterventions during follow-up (15\% planned second stage operations). Sixty percent of reinterventions were performed via endovascular approach. Acute dissection patients needed significantly fewer reinterventions $(\mathrm{n}=3 ; 8 \%)$.

Conclusions: The Thoraflex Hybrid graft adds to the frozen elephant trunk concept for treating aortic arch and descending aortic disease. Implantation of the Thoraflex Hybrid graft resulted in excellent outcomes and beneficial aortic remodeling during follow-up. This graft increases surgeons' armamentarium in the treatment of complex and diverse aortic arch pathology. ( $\mathrm{J}$ Thorac Cardiovasc Surg 2016;152:148-59)

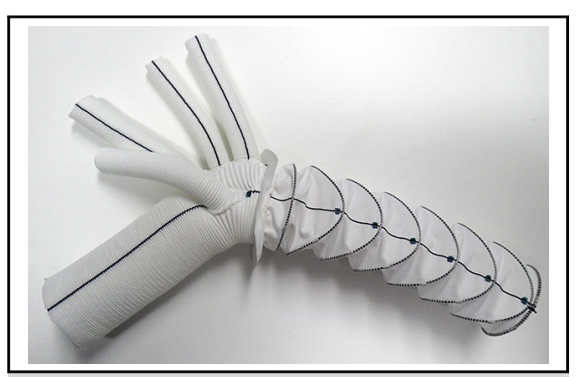

Thoraflex Hybrid graft Nascutek, Inchinnan, United Kingdom).

\section{Central Message}

Treatment of complex aortic arch disease with the Thoraflex Hybrid frozen elephant trunk graft showed excellent outcomes and aortic remodeling.

\section{Perspective}

The Thoraflex Hybrid frozen elephant trunk graft increases surgeons' armamentarium in the treatment of complex and diverse aortic arch pathology. In complicated DeBakey type I aortic dissection with malperfusion frozen elephant trunk implantation has the potential to revolutionize surgical treatment. In chronic dissection future studies have to assess the definitive role of FET treatment.

See Editorial Commentary page 160.

See Editorial page 7.
Borst and colleagues ${ }^{1}$ and others ${ }^{2,3}$ introduced the elephant trunk technique to simplify the second operation of the 2-stage procedure to treat disease of the aortic arch extending

From the Clinic for Cardiothoracic, Transplantation, and Vascular Surgery, Hannover Medical School, Hannover, Germany.

Read at the 95th Annual Meeting of The American Association for Thoracic Surgery, Seattle, Washington, April 25-29, 2015.

Received for publication May 6, 2015; revisions received Feb 9, 2016; accepted for publication Feb 26, 2016; available ahead of print May 7, 2016.

Address for reprints: Malakh Shrestha, MBBS, Clinic for Cardiothoracic, Transplantation, and Vascular Surgery, Hannover Medical School, Carl-Neuberg Strasse 1, 30625 Hannover, Germany (E-mail: shrestha.malakh.lal@mh-hannover.de).

$0022-5223 / \$ 36.00$

Copyright (c) 2016 by The American Association for Thoracic Surgery

http://dx.doi.org/10.1016/j.jtcvs.2016.02.077 into the descending aorta (aneurysms and dissection). Dake and colleagues ${ }^{4}$ introduced endovascular stent-grafts to treat aortic pathology. A combination of the above 2 techniques resulted in the frozen elephant trunk (FET) technique. Initially, various homemade FET prostheses combining conventionally available polyethylene grafts and thoracic endovascular aortic repair (TEVAR) grafts were used until prefabricated

Scanning this $\mathrm{QR}$ code will take you to a video of the AATS meeting presentation of this manuscript.

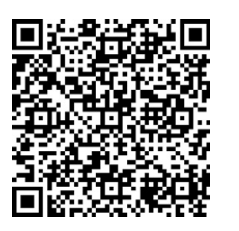



Abbreviations and Acronyms
$\mathrm{AAD}=$ acute aortic dissection
CAD = chronic aortic dissection
CSF $=$ cerebrospinal fluid
CTA = computed tomography
FET $=$ frozen elephant trunk
$\mathrm{FL} \quad=$ false lumen
HCA = hypothermic circulatory arrest
SACP = selective antegrade cerebral perfusion
TEVAR $=$ thoracic endovascular aortic repair
$\mathrm{TL} \quad=$ true lumen

hybrid prostheses became available..$^{5-8}$ The efforts culminated in the development of a prefabricated branched FET prosthesis called the Thoraflex Hybrid graft (Vascutek, Inchinnan, United Kingdom). ${ }^{9}$

The purpose of this study was to assess the perioperative and midterm results of our initial 100 patients that received the Thoraflex Hybrid FET graft for the treatment of combined diseases of the aortic arch and the descending aorta.

\section{METHODS}

\section{Description of the Graft}

The Thoraflex Hybrid graft consists of a 4-branched arch graft with a stent-graft at the distal end (Figure 1). The proximal part is a conventional gelatin-coated woven polyester prosthesis. The stented section is a self-expanding endoprosthesis constructed of thin-walled polyester and nitinol ring stents, which are attached to the fabric with braided polyester sutures. The grafts are available in different sizes (26$40 \mathrm{~mm}$ in diameter for the stented portion). The length of the stented part is either $100 \mathrm{~mm}$ or $150 \mathrm{~mm}$. Another unique feature of this graft is that the proximal unstented and distal stented parts are available in different sizes.

\section{Patients}

A synopsis of the study cohort and follow-up is shown in Figure E1. Between April 2010 and October 2014, 100 patients (65 men aged $59 \pm 14$ years) with pathologies involving the aortic arch and descending aorta underwent operation. Thirty-seven underwent acute aortic dissections (AAD group; $\mathrm{n}=36$ AAD DeBakey type $\mathrm{I}, \mathrm{n}=1$ AAD DeBakey type 3 with aortic arch dilatation), 31 underwent chronic aortic dissections (CAD group; $\mathrm{n}=24$ CAD type $\mathrm{A}, \mathrm{n}=7 \mathrm{CAD}$ type $\mathrm{B}$ ), and 32 aneurysms (aneurysm group). Informed consent was obtained from all patients. The institutional ethics committee gave approval for the study. Detailed preoperative data are given in Table 1.

\section{Surgical Technique}

After median sternotomy, extracorporeal circulation was initiated. The technique of cannulating the ascending aorta in acute dissections has been published by our group. ${ }^{10}$ The left side of the heart was vented through the right superior pulmonary vein. Blood cardioplegia was used for myocardial protection.

Replacement of the aortic arch was performed under moderate hypothermic $\left(20^{\circ} \mathrm{C}-28^{\circ} \mathrm{C}\right)^{11}$ circulatory arrest (HCA) and selective antegrade cerebral perfusion (SACP). During cooling, concomitant cardiac procedures were performed. After the patient was cooled to the desired temperature, the systemic circulation was arrested and the aortic arch opened. Catheters (Medtronic DPL, Minneapolis, Minn) were introduced into the left carotid artery and the innominate artery for SACP. The subclavian artery was clamped or occluded with a Fogarty catheter (Baxter Healthcare Group, Santa Ana, Calif).

Cerebral perfusion was initiated at a rate of $10 \mathrm{~mL} / \mathrm{kg} / \mathrm{min}$ with a pressure limit of $80 \mathrm{~mm} \mathrm{Hg}$. The blood temperature of SACP was kept $2{ }^{\circ} \mathrm{C}$ lower than HCA temperature.

The aortic arch was transected distally and the compacted endoprosthesis was deployed into the descending aorta. Thoraflex stent size was determined according to the total aortic diameter at the landing zone in all cases. In aortic aneurysms we performed an oversizing of $10 \%$. In aortic dissections and patients with Marfan syndrome no oversizing was performed. FET length was selected based on the intended landing zone. More recently, AAD and CAD were only treated with $100-\mathrm{mm}$ grafts.

A sewing collar between the graft segments simplified the distal anastomosis (Figure 1).

After the distal anastomosis was completed the perfusion to the lower body was restarted via the fourth branch of the graft. The left subclavian artery was anastomosed to the third branch of the graft and subsequently reperfused (Figure 1, $B$ ). In all patients it was possible to attach the third branch to the left subclavian artery. In selected patients this anastomosis was also performed during HCA. The proximal end of the hybrid graft was anastomosed, either to the native ascending aorta or the ascending aortic graft. After deairing the heart, coronary circulation was started again. The first and the second branch of the graft were anastomosed to the innominate and the left carotid artery. The patient was rewarmed and once cardiopulmonary bypass was discontinued, the fourth branch used for antegrade perfusion was ligated and resected. The intraoperative data are given in Table 1.

\section{Evolution of Surgical Technique}

Starting in 2013, we initiated several changes in perioperative management.

\section{Reduction of perioperative air embolism}

Use of carbon dioxide sufflation in the operative field. We used a wallmounted carbon dioxide supply. Carbon dioxide sufflation was performed at a rate of $31 / \mathrm{min}$.

\section{Myocardial protection}

To minimize cardiac ischemia time, we initiated the beating heart aortic arch surgery technique as routine procedure. ${ }^{12}$ During cooling aortic root and concomitant cardiac procedures were completed.

Subsequently the left ventricle was de-aired and the heart perfused antegradely via an aortic root cannula. Both perfusion pressure and flow were continuously monitored. Myocardial perfusion pressure was kept at 70 to $80 \mathrm{~mm} \mathrm{Hg}$ corresponding to a myocardial perfusion flow of at least 150 to $200 \mathrm{~mL} / \mathrm{min}$. The left atrium was vented through the upper right pulmonary vein. The aortic arch was then replaced on an empty beating heart. ${ }^{12}$

3. Spinal cord protection

HCA temperature was kept below $26^{\circ} \mathrm{C}$ and perioperative cerebrospinal fluid (CSF) drainage was performed $(12 \mathrm{~mm} \mathrm{Hg})$. AAD patients received CSF drainage postoperatively. In $\mathrm{AAD}$ and $\mathrm{CAD}$ patients only $100-\mathrm{mm}$ hybrid grafts were used to cover less intercostal arteries.

\section{Shortening of the HCA}

We restarted the perfusion of the lower body and started rewarming after the distal aortic arch anastomosis.

\section{Endoscopic control after deployment of FET}

In the beginning we performed endoscopic control of the FET in every patient. There was no failure of deployment. Endoscopic control was performed in selected cases only to reduce HCA time. 


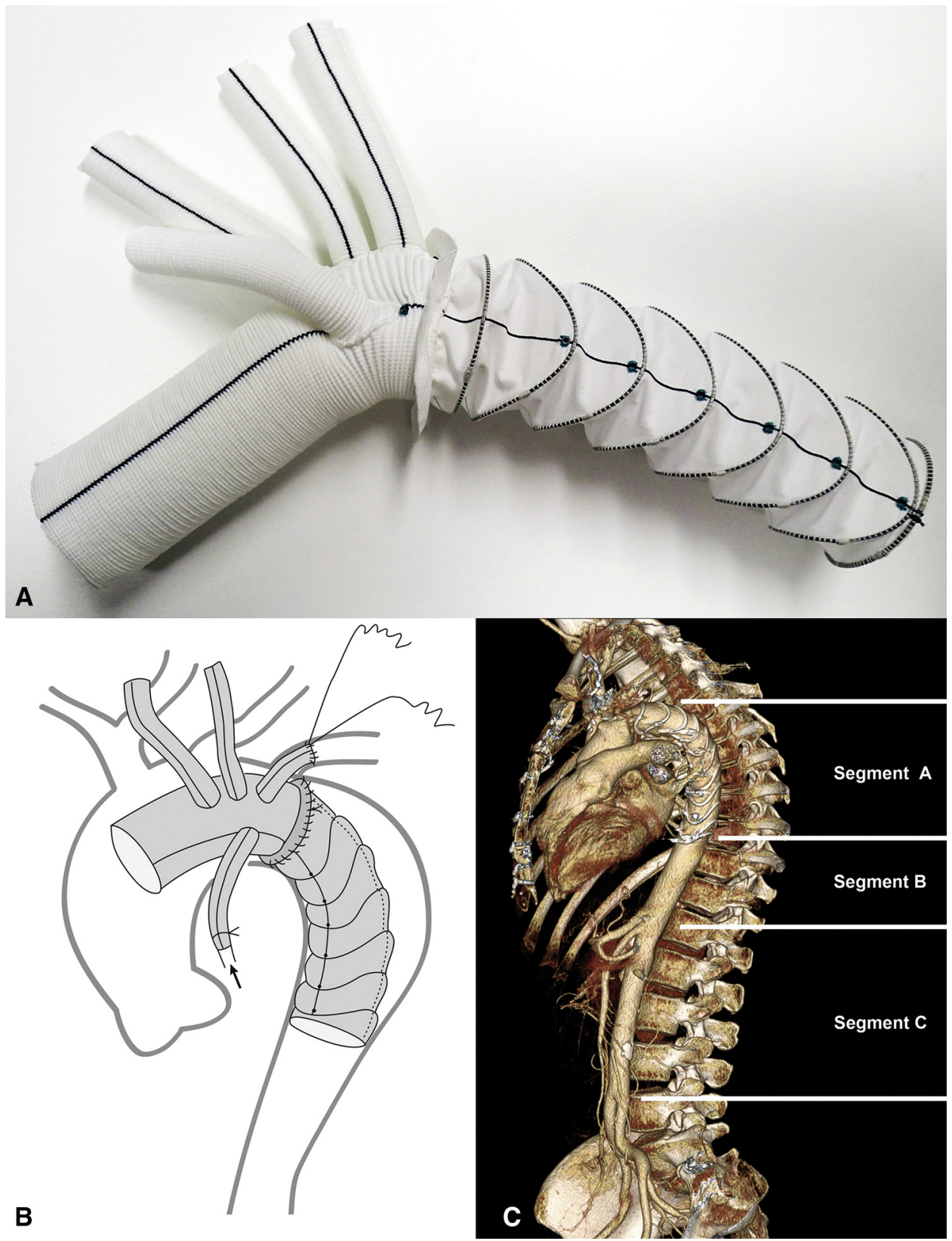

FIGURE 1. Thoraflex Hybrid graft (Vascutek, Inchinnan, Scotland). A, Deployed graft, containing 3 side branches for supra-aortic vessels, 1 sidearm for cardiopulmonary bypass, a sewing collar, and a self-expanding stented portion with nitinol ring stents. B, Illustration showing the graft during implantation. After distal aortic repair, reperfusion of the lower body is commenced. Supra-aortic vessels are reimplanted during reperfusion. C, Three-dimensional computed tomography angiography after implantation of a Thoraflex Hybrid graft in a patient with acute aortic dissection. The segments for computed tomography angiography assessment are shown: Segment A, stented part of the aorta; Segment B, downstream segment up to the Celiac trunk; and Segment $\mathrm{C}$, below the celiac trunk.

\section{Aortic Imaging}

Patients received computed tomography angiograms (CTAs) from the neck vessels to the iliac or femoral arteries preoperatively, before discharge, and during follow-up according to current guidelines. In our hospital the CTA protocol routinely involves an early arterial phase scan and a late-phase scan. All but 1 patient underwent postoperative CTA. From 93 patients who were discharged alive, 18 (19\%) died, refused follow-up CTA, or had contraindications to undergo CTA. A total of 348 CTAs were analyzed for this study.
For analysis of aortic remodeling, the descending aorta was divided into 3 segments (Figure 1,C) in line with previous work on aortic remodeling after FET placement ${ }^{13}$ : Segment A was defined as the portion from the distal aortic arch anastomosis up to the landing zone of the hybrid graft. Segment B was defined as the part distal to the hybrid graft up to the celiac trunk. Segment C was defined as the portion distal to the celiac trunk. Within each segment the maximum total aortic diameter was identified. At this level total aortic diameter, true lumen (TL), and the false lumen (FL) diameter were measured using Visage 7.1 Imaging Software 
TABLE 1. Pre-, intra-, and postoperative data

\begin{tabular}{|c|c|c|c|c|c|}
\hline & All & Acute dissection & Chronic dissection & Aneurysm & $P$ value \\
\hline \multicolumn{6}{|l|}{ Preoperative data } \\
\hline Patients & 100 & 37 & 31 & 32 & \\
\hline Male sex & $65(65 \%)$ & $28(76 \%)$ & $20(65 \%)$ & $17(53 \%)$ & .15 \\
\hline Age, y & $62(49-70)$ & $55(47-68)$ & $53(43-67)$ & $68(61-75)$ & .0001 \\
\hline Marfan syndrome & $12(12 \%)$ & $5(14 \%)$ & $7(23 \%)$ & $0(0 \%)$ & .021 \\
\hline Previous surgery & $28(28 \%)$ & $1(3 \%)$ & $23(74 \%)$ & $4(13 \%)$ & $<.0001$ \\
\hline Renal insufficiency & $17(17 \%)$ & $5(14 \%)$ & $6(19 \%)$ & $6(19 \%)$ & .77 \\
\hline Malperfusion & $11(11 \%)$ & $10(27 \%)$ & $1(3 \%)$ & $0(0 \%)$ & .0004 \\
\hline \multicolumn{6}{|l|}{ Operative data } \\
\hline Operation time, $\min$ & $367 \pm 80$ & $391 \pm 62$ & $398 \pm 90$ & $310 \pm 57$ & $<.0001$ \\
\hline Cardiopulmonary bypass time, $\min$ & $243 \pm 61$ & $267 \pm 46$ & $250 \pm 67$ & $206 \pm 54$ & $<.0001$ \\
\hline Cardiac ischemia time, $\min$ & $101 \pm 65$ & $130 \pm 60$ & $95 \pm 65$ & $75 \pm 59$ & .013 \\
\hline Distal HCA time, min & $47(36-61)$ & $47(36-64)$ & $58(44-72)$ & $40(34-54)$ & .0034 \\
\hline HCA temperature, ${ }^{\circ} \mathrm{C}$ & $25(24-26)$ & $25(22-26)$ & $25(24-26)$ & $25(25-26)$ & .47 \\
\hline SACP time, $\min$ & $98(77-115)$ & $104(89-132)$ & $102(87-118)$ & $84(57-100)$ & .0002 \\
\hline Beating heart arch surgery* & $47(47 \%)$ & $14(38 \%)$ & $16(52 \%)$ & $17(53 \%)$ & .37 \\
\hline Concomitant procedures & $54(54 \%)$ & $27(73 \%)$ & $14(45 \%)$ & $13(42 \%)$ & .017 \\
\hline Bentall & 11 & 5 & 5 & 1 & \\
\hline Valve sparing root & 30 & 21 & 4 & 5 & \\
\hline $\mathrm{CABG}$ & 12 & 4 & 2 & 6 & \\
\hline MVR & 3 & 1 & 1 & 1 & \\
\hline AVR & 3 & 0 & 1 & 2 & \\
\hline Other & 3 & 1 & 2 & 0 & \\
\hline \multicolumn{6}{|l|}{ Intraoperative blood products } \\
\hline Packed RBC, U & $6.0(4.0-8.0)$ & $6.0(5.5-10)$ & $6.0(3.5-9.0)$ & $5.0(3.8-6.0)$ & .024 \\
\hline Platelets, U & $3.0(2.0-4.0)$ & $4.0(3.0-4.0)$ & $3.0(2.0-4.0)$ & $3.0(2.0-4.0)$ & .051 \\
\hline FFP, U & $6.0(4.0-8.0)$ & $6.0(6.0-10)$ & $6.0(6.0-10)$ & $4.5(4.0-6.0)$ & .002 \\
\hline \multicolumn{6}{|l|}{ Postoperative data } \\
\hline Ventilation, d & $1.0(0.6-3.7)$ & $2.8(0.8-6.0)$ & $0.9(0.5-3.7)$ & $0.7(0.5-1.1)$ & .0014 \\
\hline Prolonged ventilation & $29(29 \%)$ & $16(43 \%)$ & $9(29 \%)$ & $4(13 \%)$ & .020 \\
\hline Rethoracotomy for bleeding & $10(10 \%)$ & $6(16 \%)$ & $3(10 \%)$ & $1(3 \%)$ & .19 \\
\hline Stroke & $9(9 \%)$ & $5(14 \%)$ & $3(10 \%)$ & $1(3 \%)$ & .32 \\
\hline Paraparesis & $7(7 \%)$ & $3(8 \%)$ & $2(7 \%)$ & $2(6 \%)$ & .96 \\
\hline Recurrent nerve palsy & $25(25 \%)$ & $8(22 \%)$ & $12(39 \%)$ & $5(14 \%)$ & .049 \\
\hline Acute kidney injury & $30(30 \%)$ & $12(32 \%)$ & $12(39 \%)$ & $6(20 \%)$ & .27 \\
\hline Dialysis & $14(14 \%)$ & $4(11 \%)$ & $7(23 \%)$ & $3(9 \%)$ & .25 \\
\hline Dialysis at discharge & $8(8 \%)$ & $3(8 \%)$ & $3(10 \%)$ & $2(6 \%)$ & .88 \\
\hline Hospital stay, d & $17(12-27)$ & $16(11-27)$ & $20(15-31)$ & $15(11-22)$ & .13 \\
\hline Perioperative mortality & $7(7 \%)$ & $3(8 \%)$ & $1(3 \%)$ & $3(9 \%)$ & .60 \\
\hline \multicolumn{6}{|l|}{ Follow-up data } \\
\hline Patients in follow-up & $93(93 \%)$ & $34(92 \%)$ & $30(97 \%)$ & $29(91 \%)$ & \\
\hline Follow-up, y & $2.7(1.8-4.6)$ & $2.7(2.0-4.7)$ & $2.7(1.7-4.5)$ & $2.5(1.7-4.6)$ & .52 \\
\hline Death after discharge & $12(13 \%)$ & $3(9 \%)$ & $4(13 \%)$ & $5(17 \%)$ & .99 \\
\hline Aortic reoperation & $22(22 \%)$ & $3(8 \%)$ & $9(29 \%)$ & $10(31 \%)$ & .036 \\
\hline Time to aortic reoperation, mo & $7.3 \pm 5.3$ & $8.6 \pm 7.8$ & $8.3 \pm 3.8$ & $5.5 \pm 5.9$ & .47 \\
\hline Open surgical & $9(9 \%)$ & $1(3 \%)$ & $4(13 \%)$ & $4(13 \%)$ & .24 \\
\hline Thoracoabdominal & 2 & 0 & 0 & 2 & \\
\hline Descending aorta & 3 & 1 & 2 & 0 & \\
\hline Abdominal/iliac & 2 & 0 & 2 & 0 & \\
\hline Other & 2 & 0 & 0 & 2 & \\
\hline Endovascular & $13(13 \%)$ & $2(5 \%)$ & $5(16 \%)$ & $6(19 \%)$ & .21 \\
\hline Follow-up CTA & $75(81 \%)$ & $28(82 \%)$ & $26(87 \%)$ & $21(72 \%)$ & .36 \\
\hline
\end{tabular}

Values are presented as n (\%), median and interquartile range (quartile 1-quartile 3), mean \pm standard deviation, or $\mathrm{n}$. $H C A$, Hypothermic circulatory arrest; SACP, selective antegrade cerebral perfusion; $C A B G$, coronary artery bypass grafting; $M V R$, mitral valve repair/replacement; $A V R$, aortic valve replacement; $R B C$, red blood cells; $F F P$, fresh frozen plasma; CTA, computed tomography angiography. *Beating heart arch surgery refers to noncardioplegic, continuous myocardial perfusion during aortic arch repair (see Methods). 
(Visage Imaging GmbH, Berlin, Germany). FL thrombosis was analyzed for each segment after volumetric reconstruction and was classified as either patent $(<20 \%$ thrombosis of FL), partially thrombosed $(20 \%$ $80 \%$ thrombosis), or thrombosed ( $>80 \%$ thrombosed or obliterated). Aortic remodeling was classified as either positive (aortic diameter reduction $>10 \%$ or stable aortic diameter $[ \pm 10 \%]$ with increase of TL diameter increase of $\geq 10 \%$ ), stable (changes within $\pm 10 \%$ ), or negative (all other). Endoleaks were assessed according to published recommendations for aortic endovascular therapy. ${ }^{14}$

\section{Statistical Analysis}

Clinical data of the initial 57 patients were collected prospectively. Of these patients 34 were part of the CE certification trial and 19 patients were part of the prospective postmarket registry. Clinical data of the following 43 patients and all aortic remodeling data were analyzed retrospectively. GraphPad Prism version 6.1 for Windows (La Jolla, Calif) and IBM-SPSS Statistics version 23 (Armonk, NY) were used. Continuous variables were given as mean \pm standard deviation or median and interquartile range (quartile 1-quartile 3). D'Agostino and Pearson omnibus normality test was performed to check for normal distribution. Categorical variables were summarized as total number and percentages. We used $\chi^{2}$ or Fisher exact test to analyze differences of dichotomous variables, as appropriate. The unpaired $t$ test or the nonparametric Mann-Whitney $U$ test were used for group comparisons of continuous variables. One-way analysis of variance and Tukey test or KruskalWallis and Dunn test were used for multiple group comparisons of continuous variables. The Kaplan-Meier survival estimate was used to analyze survival, freedom from aortic reoperations, and thrombosis of FL in aortic dissections. Statistical differences in Kaplan-Meier survival were determined with the log-rank test. The procedure linear mixed models was applied to analyze time course developments of diameters and to detect different developments between disease entities. All comparisons were unadjusted.

\section{RESULTS}

The detailed clinical data are given in Table 1. The Thoraflex Hybrid prosthesis could be deployed in all cases without technical failure. Aneurysm patients were significantly older than AAD and CAD patients, received fewer concomitant procedures, had shorter operation times, and needed fewer blood transfusions. In elective patients, the mean ventilation time was $<1$ day. A total of 7 patients (7\%) experienced paraparesis. Three were AAD patients who had received $150-\mathrm{mm}$ stent grafts and were not routinely treated with CSF drainage after the operation. All but 1 recovered with CSF drainage.

A total of 14 patients (14\%) experienced acute renal failure requiring dialysis. Four were AAD patients. Eight patients $(8 \%)$ were discharged on dialysis.

There were 7 perioperative deaths $(7 \% ; 30$-day mortality and in-hospital deaths). The cause of deaths were lung embolism $(\mathrm{n}=1)$, intracerebral bleeding $(\mathrm{n}=1)$, cardiac failure $(n=2)$, sepsis $(n=1)$, cerebral edema $(n=1)$, and stroke $(n=1)$. Of these patients, 3 presented with $\mathrm{AAD}$ and 2 with malperfusion. In both patients who died due to cardiac failure, beating heart aortic arch surgery technique $^{12}$ was not used.

Follow-up was $100 \%$ complete and is summarized in Figure E1 and Table 1. Kaplan-Meier curves for survival and aortic reoperation are shown in Figure 2. Aortic diameters during follow-up are given in Figure 3. FL thrombosis rate in $\mathrm{AAD}$ and $\mathrm{CAD}$ patients and the rate of endoleaks in aneurysm patients are shown in Figure 4. The degree of aortic remodeling during follow-up is shown in Figure 5.

Secondary reintervention on the downstream aorta was more common in aneurysm and CAD patients compared with AAD patients (Figure 2, D, and Table 1). Twenty-two percent of patients received treatment of the downstream aorta during follow-up, the majority being anticipated second procedures (15 out of 22 [68\%]). Fifty-nine percent of aortic reinterventions (13 out of 22 patients) were done by an endovascular approach. Overall midterm survival did not differ significantly between groups (Figure 2).

In AAD patients total aortic diameters in all 3 aortic segments were significantly smaller compared with CAD or aneurysm patients (Figure 3). TL diameters were similar in AAD and CAD. In stented segment A Thoraflex FET implantation resulted in a significant increase of TL diameters and stable total aortic diameters during follow-up. This led to a high rate of positive aortic remodeling (Figure 4). The effect remained stable during follow-up. Thoraflex FET implantation positively affected TL diameters in downstream segment B during follow-up. However, due to increasing total aortic diameters, neither in segment $\mathrm{B}$ nor in segment $\mathrm{C}$ aortic remodeling remained stable in AAD patients. The incidence of negative aortic remodeling significantly increased during follow-up FL.

In CAD patients, Thoraflex FET implantation also resulted in an increase of TL diameters and decrease of FL diameters in stented segment A with a high rate of stable or positive aortic remodeling (Figures 3 and 4). In contrast to AAD patients, total aortic diameters significantly decreased during follow-up in CAD patients, predominantly due to a more pronounced decrease of FL diameters. The positive downstream effect of Thoraflex FET implantation on TL diameters in AAD patients in segment $B$ could not be shown in CAD patients. TL in both AAD and CAD patients Thoraflex FET implantation resulted in a $100 \%$ FL thrombosis rate in stented segment A within 24 months (Figure 5). The thrombosis rate was significantly faster in AAD patients compared with CAD patients. However, the effect of Thoraflex FET implantation on FL thrombosis in downstream segments B and C was low.

Sixty-three percent of aneurysm patients (20 out of 32) had an aortic diameter in segment B larger than $40 \mathrm{~mm}$ (largest FET size). As expected, $41 \%$ of patients (13 out of 32) showed an endoleak Ib postoperatively (Figure 5, D). Overall rate of positive aortic remodeling after Thoraflex FET treatment in aortic aneurysm patients was lower than in AAD and CAD patients (Figure 4). 

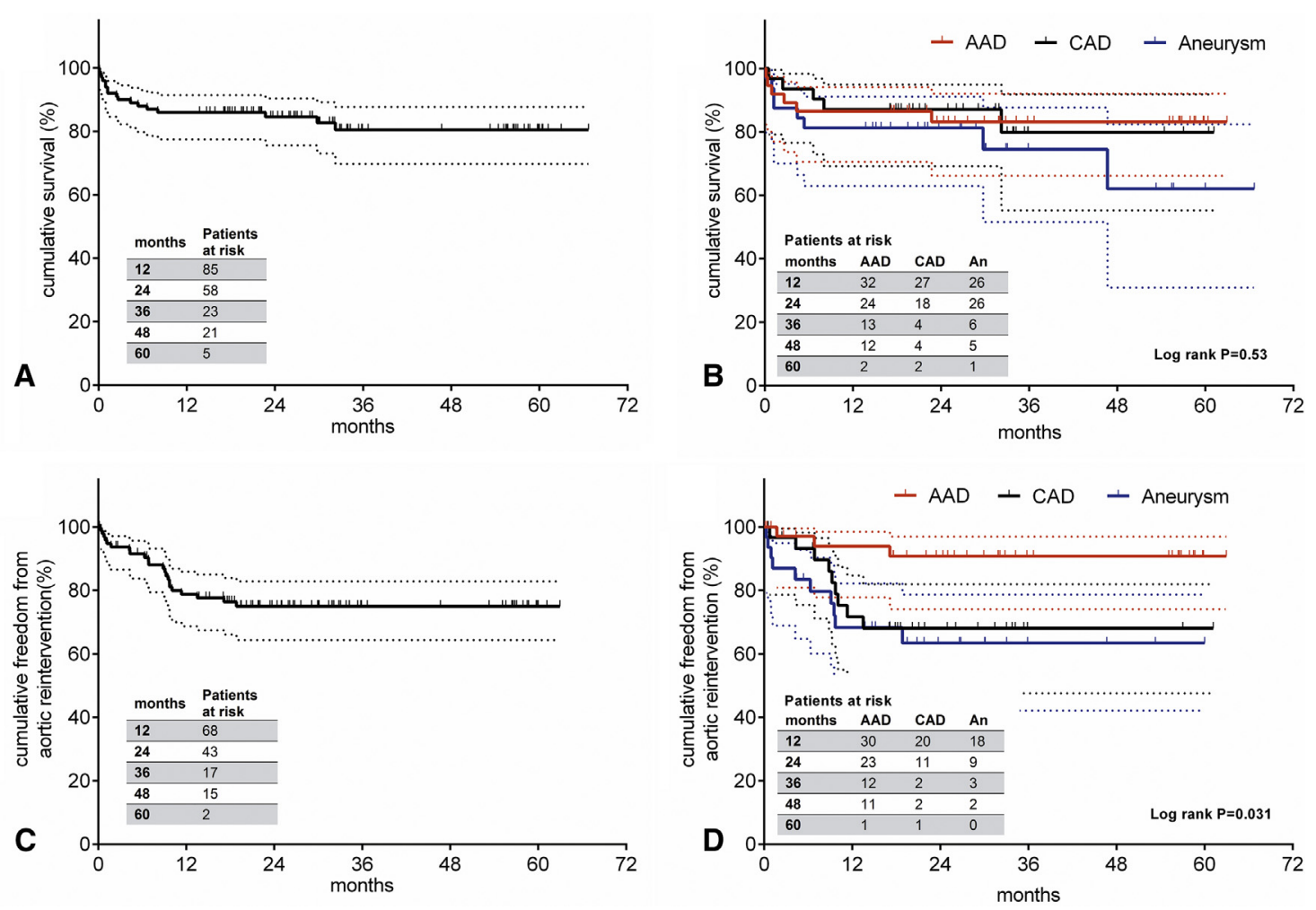

FIGURE 2. Kaplan-Meier curves for survival and freedom of aortic reoperation. A, Survival, total patient cohort. B, Survival, acute aortic dissection ( $A A D)$ versus chronic aortic dissection $(C A D)$ versus aneurysm $(A n)$ group. C, Freedom from aortic reoperation, total group. D, Freedom from aortic reoperation, $\mathrm{AAD}$ versus $\mathrm{CAD}$ versus aneurysm group.

\section{DISCUSSION}

The Thoraflex Hybrid graft combines the advantages of FET treatment with that of the branched plexus graft for the aortic arch and the supra-aortic vessels. Our experience with the first 100 implantations of Thoraflex Hybrid graft shows that the graft is technically easy to implant, and that graft function is excellent during early and midterm follow-up.

\section{AAD (DeBakey Type I)}

The implantation of a FET in DeBakey type I acute dissection may be helpful because in patients with malperfusion, total aortic arch replacement with FET implantation treats all entries that may exist in the transverse aortic arch, expands the TL, and depressurizes the FL in the proximal descending aorta. FET can prevent future aortic events (eg, aneurysm formation in the chronically dissected descending aorta).

In uncomplicated $\mathrm{AAD}$, the decision to proceed with FET implantation has to balance the risk of a more demanding radical procedure against the mid- and long-term benefits. Prevention of postdissection aneurysm formation is attractive because secondary surgical repair may be technically challenging and secondary TEVAR repair is not always possible, especially with a remaining dissection of the transverse aortic arch.

In case of lower body malperfusion in AAD patients, total aortic arch replacement with FET implantation in our view improves outcome. In our series 10 AAD patients presented with visceral and/or lower body malperfusion. Only 1 patient died due to neurologic complications. The perioperative mortality in AAD patients was $8 \%$ in our series and compares favorably with other studies. ${ }^{15,16}$ Due to our positive experience we do not delay surgical correction of AAD with malperfusion. ${ }^{17}$

During follow-up AAD patients showed early thrombosis of the FL and a high rate of positive remodeling of the descending aorta in the stented segment. Hence, the FET procedure prevents further operations in the treated segment. One has to remember that 24 patients $(24 \%)$ in this series underwent reoperation for adverse aortic remodeling of the aortic arch and proximal descending aorta after limited repair of AAD. These patients would not have needed this operation if they had received FET in the initial operation.

Future studies have to clarify whether FET implantation has a beneficial effect on the aortic reoperation rate of the nontreated downstream aorta. 
Segment A
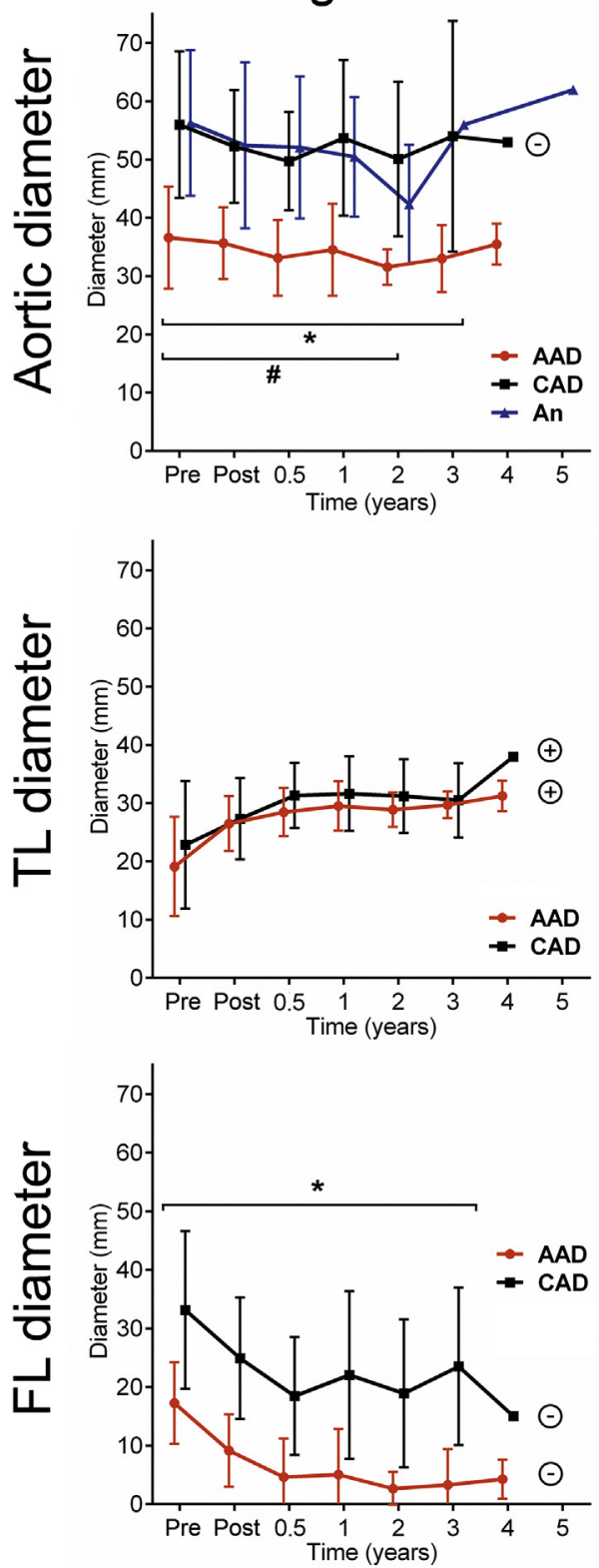

Segment B
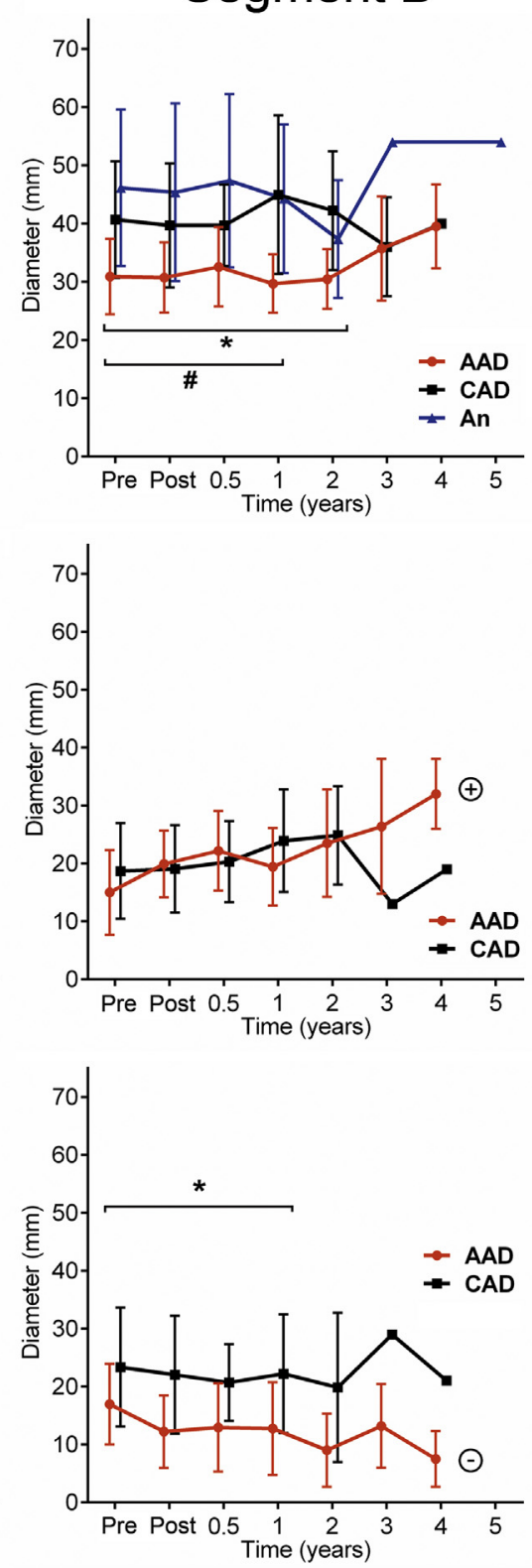

Segment C
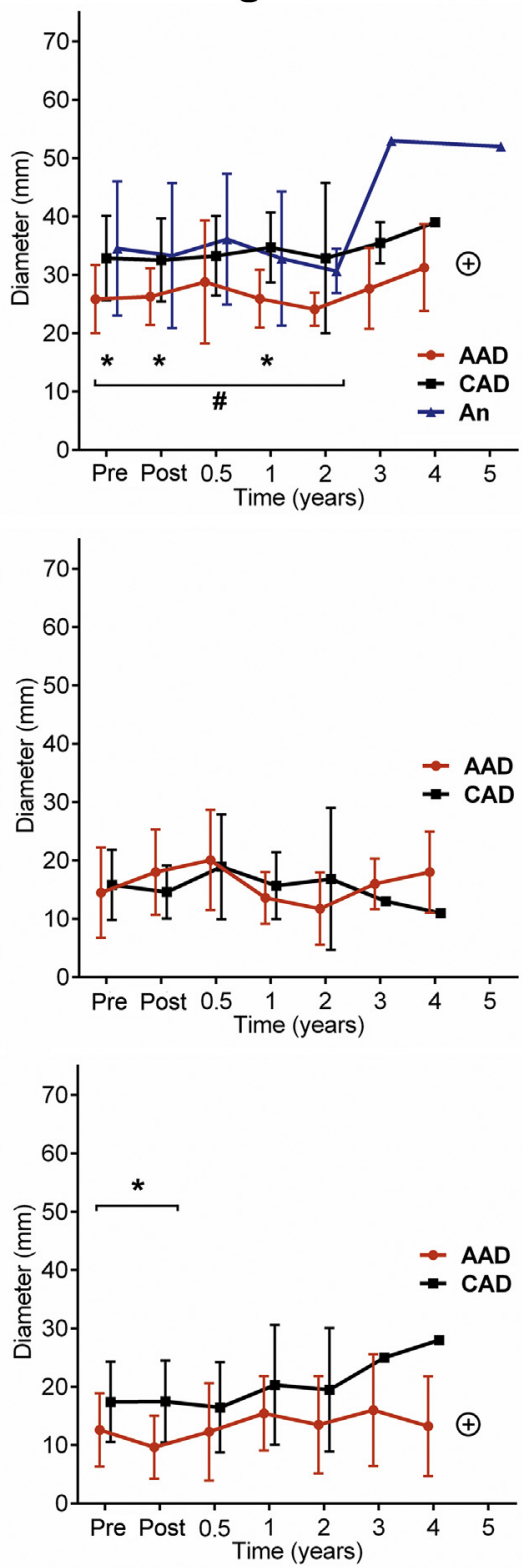

FIGURE 3. Changes of aortic diameters after frozen elephant trunk treatment with Thoraflex Hybrid graft (Vascutek, Inchinnan, Scotland). Aortic diameters according to measurements of the largest part of each segment in computed tomography angiography (CTA) during follow-up. Time points were defined as preoperative (pre), postoperative; that is, discharge CTA (post) or according to time after surgery (years). Segments A, B, and C of the descending aorta are classified as depicted in Figure 1 and shown in columns. Top row shows total aortic diameters. Middle row shows TL diameters. Bottom row shows FL diameters. Diameter changes over time were assessed by linear mixed models analysis. $A A D$, Acute aortic dissection; $C A D$, chronic aortic dissection; $A n$, aneurysm; $T L$, true lumen; $F L$, false lumen. ${ }^{*} P<.05$, AAD versus CAD. $\# P<.05$ versus An. + Significant increase during follow-up, $(P<.05)$. - Significant decrease during follow-up $(P<.05)$.

In summary, our findings confirm the notion of positive post-FET remodeling of an acutely dissected aorta and reduction of secondary aortic procedures.

Our experience shows that in patients with connective tissue disease it is important to avoid oversizing of the FET.

\section{CAD}

When working with CAD patients surgeons should carefully assess for the presence of reentries in the downstream aorta. This helps to minimize the risks of malperfusion due to thrombosis of the FL. Ideally, a completion angiography would confirm the absence of 
Segment A
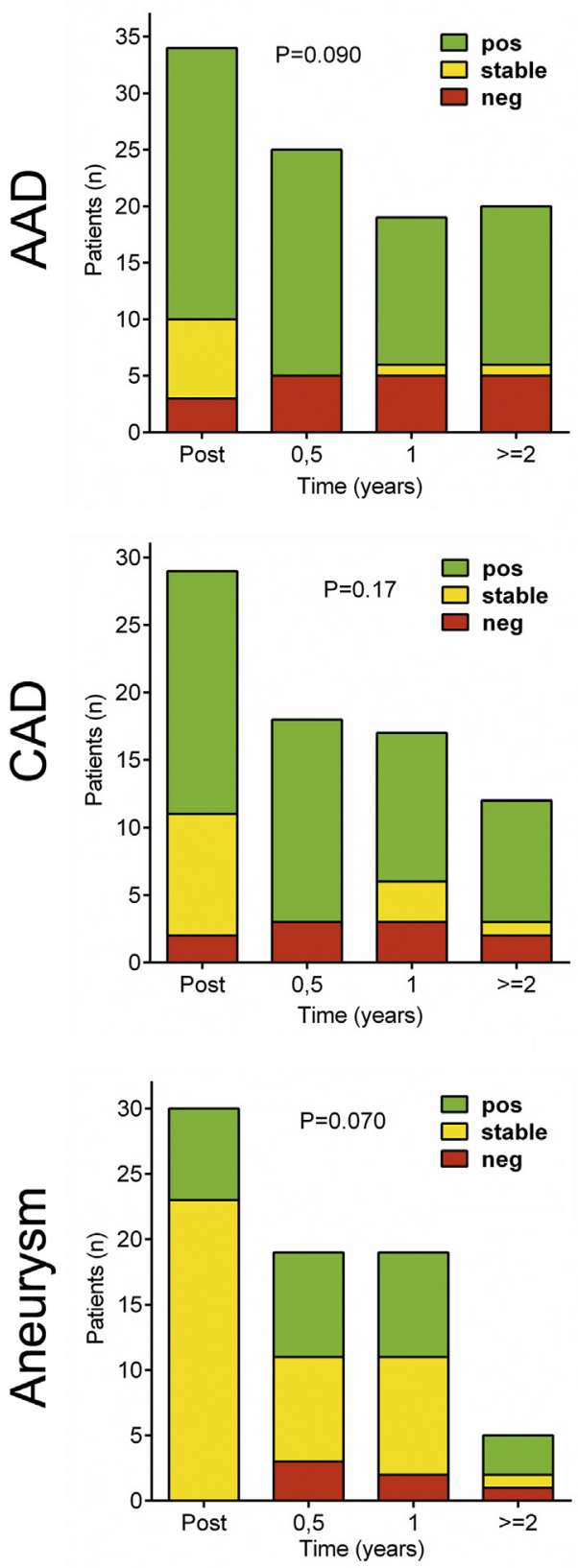

Segment B
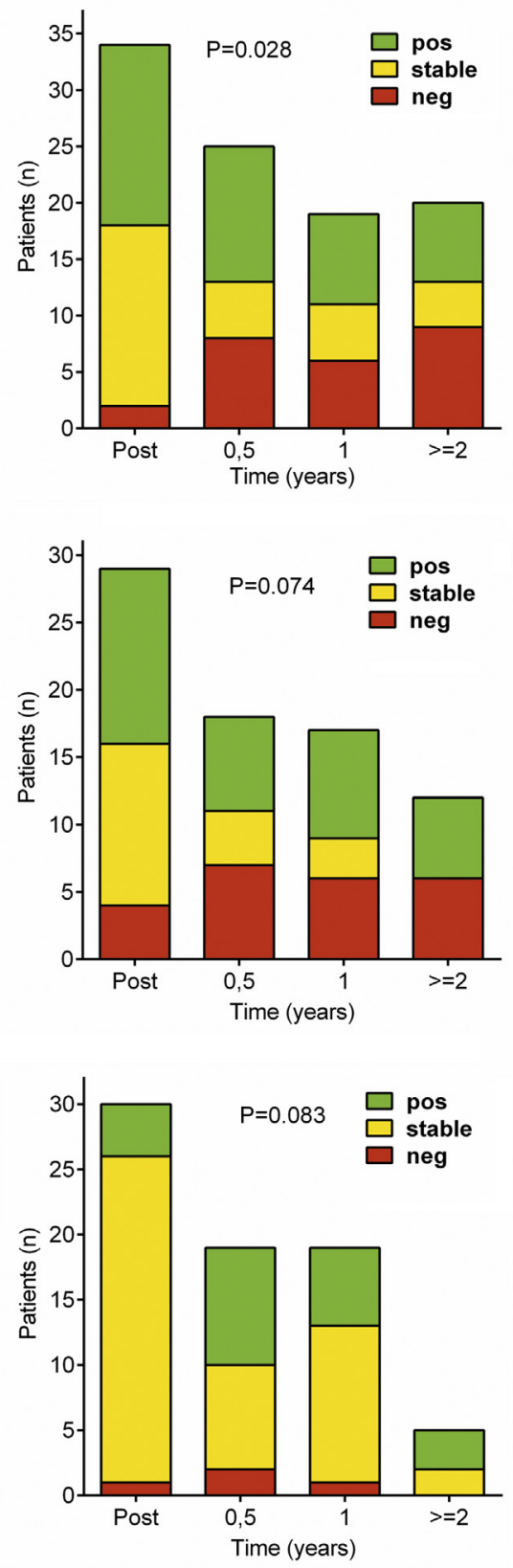

Segment C
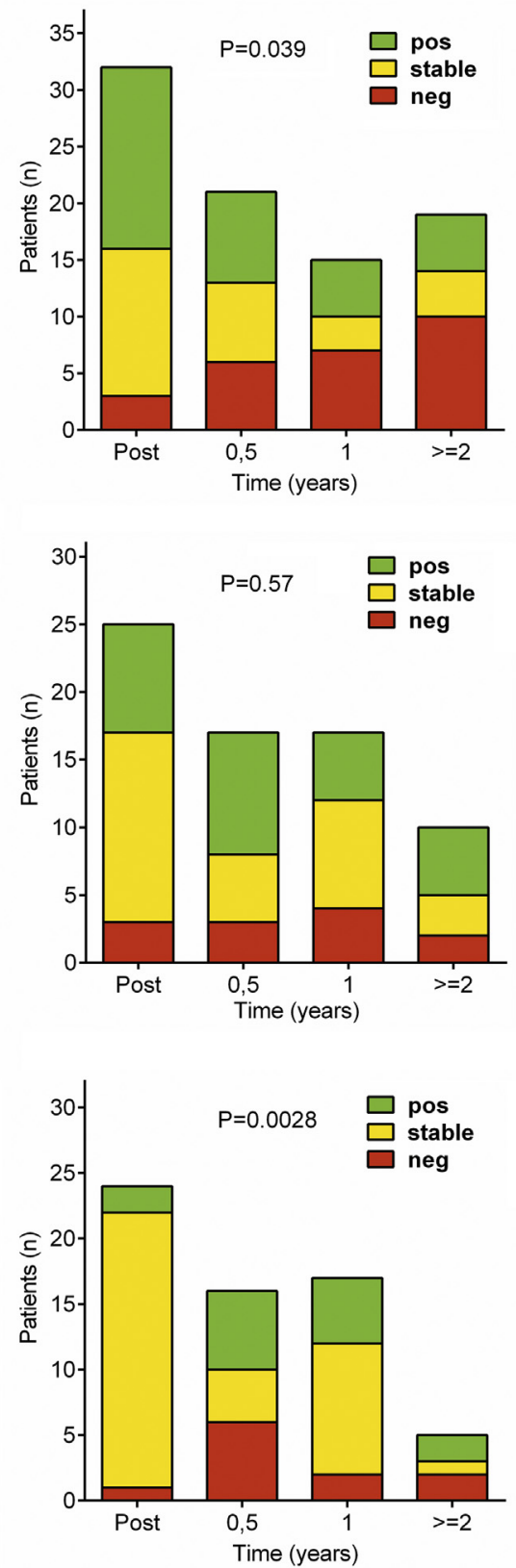

FIGURE 4. Aortic remodeling after frozen elephant trunk treatment with Thoraflex Hybrid graft (Vascutek, Inchinnan, Scotland). Based on individual patient's changes of total aortic diameter, true lumen diameter, and false lumen diameter, aortic remodeling is assessed and defined for each disease and aortic segment as positive (pos), negative (neg), or stable. Segments A, B, and C of the descending aorta are classified as depicted in Figure 1 and shown in columns. Top row shows aortic remodeling in acute aortic dissection $(A A D)$ cases, middle row shows chronic aortic dissection $(C A D)$ cases, bottom row aneurysm cases. $P$ value calculations give results from $\chi^{2}$ test within each segment/disease data set. Due to small numbers, computed tomography angiography from 2 - to 5-year follow-up had to be summarized. Columns show absolute numbers of computed tomography angiography scans per patients assessed.

postoperative malperfusion but may increase the risk of postoperative renal failure. ${ }^{18}$ Alternatively, abdominal ultrasonography can be performed.

The results of our series show that the remodeling of the stented segment of the descending aorta takes longer in
CAD patients compared with AAD patients. Nevertheless, FL thrombosis rate is $100 \%$ within 2 years postsurgery. Although the difference was not statistically significant in this study, we believe that CAD patients have a higher risk of perioperative renal failure after FET implantation. 

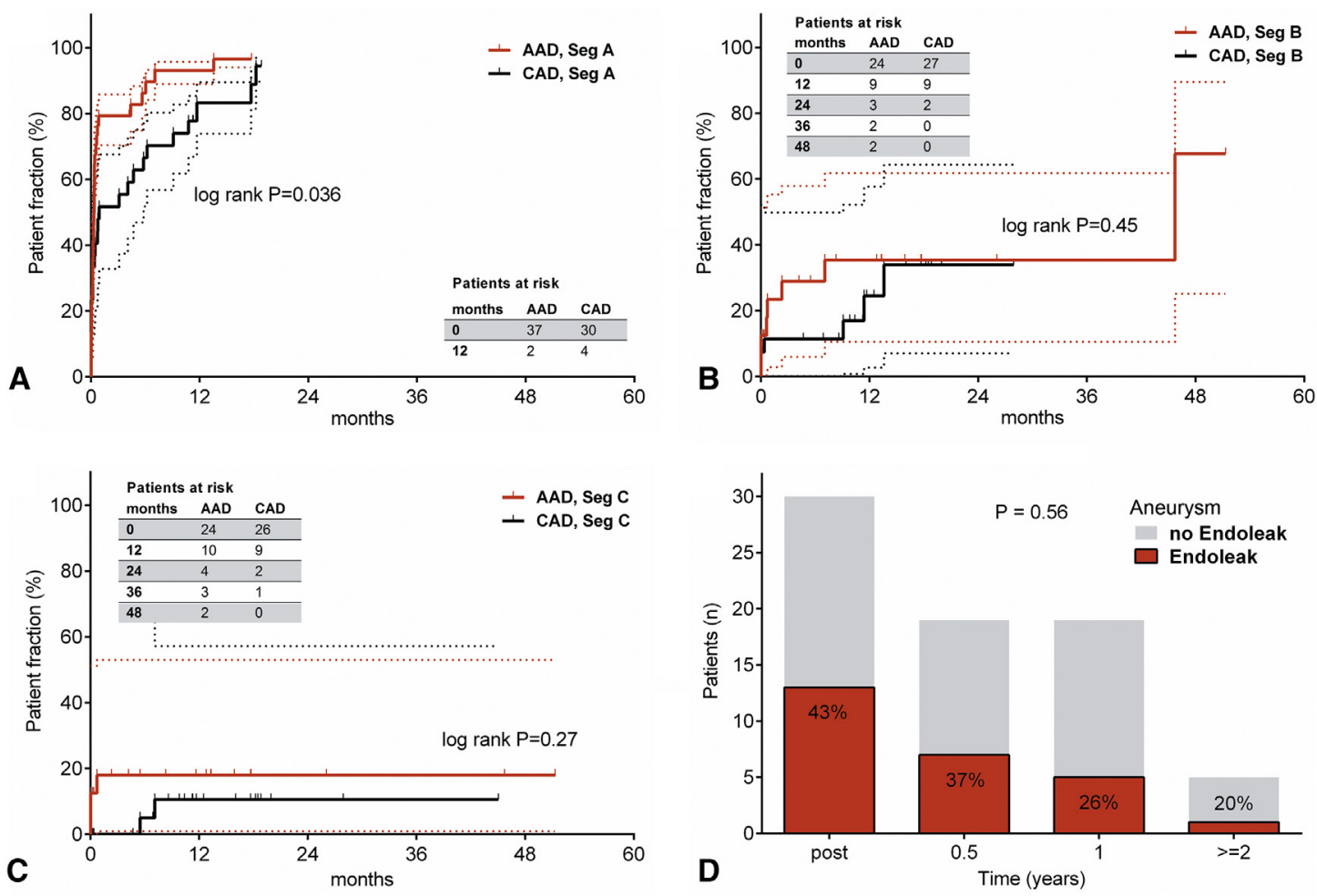

FIGURE 5. Kaplan-Meier curves of false lumen thrombosis rate in acute aortic dissection $(A A D)$ and chronic aortic dissection $(C A D)$ patients and endoleak rate in aneurysm patients after treatment with Thoraflex Hybrid graft (Vascutek, Inchinnan, Scotland). Segments A (Seg A), B (Seg B), and (Seg C) of the descending aorta are classified as depicted in Figure 1. False lumen thrombosis is defined as thrombosis of $>80 \%$ or complete obliteration of the false lumen. A, False lumen thrombosis rate of stented Seg A. Kaplan-Meier curves end at 19 months, because every patient at risk reached the end point (false lumen thrombosis). B, False lumen thrombosis rate of downstream Seg B. C, False lumen thrombosis rate of downstream Seg C. D, Columns show relative incidence of type IB endoleaks after frozen elephant trunk treatment of aneurysm patients with the Thoraflex Hybrid graft. $P$ value calculation gives result of $\chi^{2}$ test. Columns show absolute numbers of computed tomography angiography scans per patients assessed.

Further studies have to clarify the benefits and risks of FET treatment in these patients.

\section{CAD (DeBakey Type III)}

In these patients TEVAR is the treatment of first choice. However, the FET technique represents an alternative if there is no landing zone for TEVAR.

In both $\mathrm{AAD}$ and $\mathrm{CAD}$, a length of $100 \mathrm{~mm}$ for the FET seems to be enough, because its primary purpose is to stabilize the dissected membrane and to improve downstream TL expansion. When treating patients undergoing $\mathrm{CAD}$ it could be argued to implant longer FET grafts in selected patients (eg, to cover multiple reentries). Because longer FET stent grafts may result in a higher risk of spinal cord injury ${ }^{15}$ we have stopped using 150-mm Thoraflex Hybrid grafts in CAD patients.

\section{Aortic Aneurysms}

In complex aortic arch aneurym, for a true single-stage operation with FET, the disease has to be limited to the level of the distal landing zone. If patients are not selected carefully or the disease progresses into the downstream aorta, second-stage procedure is imperative even with FET. ${ }^{19}$ It remains a strategic choice whether a primary distal seal is intended or secondary TEVAR for completion is chosen. Treatment with Thoraflex Hybrid FET qualifies for both secondary open and endovascular repair. An open surgical completion is also possible should such a need arise. The modern nitinol stents of Thoraflex Hybrid FET makes clamping possible and the stent-graft resumes its former configuration after declamping.

We have implanted the Thoraflex FET in patients with aortic aneurysms that extend below the landing zone of the stent-graft intentionally as a first-stage procedure before subsequent TEVAR treatment.

\section{Spinal Cord Injury After FET Implantation}

Spinal cord injury has been described as a complication after FET implantation in up to $20 \%$ of cases in some series. ${ }^{15}$ Minimum core temperature, HCA duration, as well as FET length have been discussed as potential predictors. In the series presented here, we experienced a 
spinal cord injury rate of $7 \%$. One could argue that CAD patients are more prone to spinal cord injury if rapid FL thrombosis occurs postoperatively. We experienced this complication in 2 CAD patients. Both recovered with prolonged CSF drainage. In addition, we have reduced $\mathrm{HCA}$ temperature in these procedures to at least $25^{\circ} \mathrm{C}$ and limited the length of the stent-graft to $100 \mathrm{~mm}$ in aortic dissections. All elective patients preoperatively receive CSF drainage, whereas AAD patients receive a CSF drain as soon as possible after the operation. Because of the unique design of the Thoraflex Hybrid graft, reperfusion of the lower body can be started immediately after completion of the distal anastomosis. This may help in reducing HCA duration and the risk for spinal cord injury.

Because of the small number of patients experiencing spinal cord injury in our series and the relatively equal distribution between the groups we have not yet been able to identify specific predictors for spinal cord injury.

To reduce the perioperative risks of total aortic arch replacement with FET, we have modified our operative techniques, namely, use of carbon dioxide, perioperative CSF drainage, restarting perfusion of the myocardium as early as possible, and replacing the aortic arch on a continuously perfused heart (ie, beating heart arch surgery). ${ }^{11,12}$

It is obvious that patients after FET implantation warrant a similar follow-up protocol as patients after TEVAR. Therefore, we advise that these patients be kept under strict follow-up observation.

\section{Limitations}

The main limitation of this study is the limited follow-up time and its mostly retrospective data collection. This was a single-center experience. In addition, the observational nature of the study makes subgroup comparison difficult.

\section{CONCLUSIONS}

In selected patients with aortic aneurysm, Thoraflex Hybrid FET potentially allows for single-stage therapy. In AAD DeBakey type I, this graft stabilizes the dissecting membrane and facilitates TL expansion. This in our view is especially beneficial in patients with malperfusion.

This FET graft favors FL thrombosis in both AADs and CADs. If necessary, it offers an ideal landing zone for endovascular completion.

This graft substantially adds to the FET concept for treating the aortic arch and descending aorta. Our experience demonstrates excellent perioperative results and positive aortic remodeling during midterm follow-up. Combining the FET with a 4-branched aortic arch graft substantially increases surgeons' armamentarium in the treatment of complex aortic arch pathology.

\section{Webcast}

You can watch a Webcast of this AATS meeting presentation by going to: http://www.jtcvsonline.org/ article/S0022-5223(16)30036-8/addons.

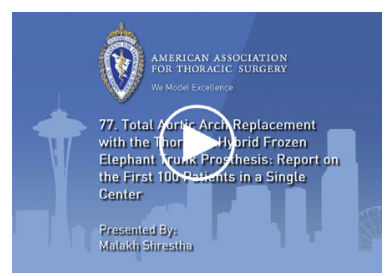

\section{Conflict of Interest Statement}

M.S. serves as a consultant for Vascutek Terumo. A.H. serves as a consultant for Vascutek Terumo and Edwards Lifesciences, and receives grant support from Thoratec. J.S. serves as a consultant for Thoratec and Heartware. All other authors have nothing to disclose with regard to commercial support.

\section{References}

1. Borst HG, Walterbusch G, Schaps D. Extensive aortic replacement using "elephant trunk" prosthesis. Thorac Cardiovasc Surg. 1983;31:37-40.

2. Crawford ES, Coselli JS, Svensson LG, Safi HJ, Hess KR. Diffuse aneurysmal disease (chronic aortic dissection, Marfan, and mega aorta syndromes) and multiple aneurysm. Treatment by subtotal and total aortic replacement emphasizing the elephant trunk operation. Ann Surg. 1990;211:521-37.

3. Svensson LG, Kim KH, Blackstone EH, Alster JM, McCarthy PM, Greenberg RK, et al. Elephant trunk procedure: newer indications and uses. Ann Thorac Surg. 2004;78:109-16.

4. Dake MD, Miller DC, Mitchell RS, Semba CP, Moore KA, Sakai T. The "first generation" of endovascular stent-grafts for patients with aneurysms of the descending thoracic aorta. J Thorac Cardiovasc Surg. 1998;116:689-703.

5. Suto Y, Yasuda K, Shiiya N, Murashita T, Kawasaki M, Imamura M, et al. Stented elephant trunk procedure for an extensive aneurysm involving distal aortic arch and descending aorta. J Thorac Cardiovasc Surg. 1996;112:1389-90.

6. Kato M, Ohnishi K, Kaneko M, Ueda T, Kishi D, Mizushima T, et al. New graft-implanting method for thoracic aortic aneurysm or dissection with a stented graft. Circulation. 1996;94(9 Suppl):II188-93.

7. Usui A, Ueda Y, Watanabe T, Kawaguchi O, Ohara Y, Takagi Y, et al. Clinical results of implantation of an endovascular covered stent-graft via midsternotomy for distal aortic arch aneurysm. Cardiovasc Surg. 2000;8:545-9.

8. Karck M, Chavan A, Hagl C, Friedrich H, Galanski M, Haverich A. The frozen elephant trunk technique: a new treatment for thoracic aortic aneurysms. J Thorac Cardiovasc Surg. 2003;125:1550-3.

9. Shrestha M, Pichlmaier M, Martens A, Hagl C, Khaladj N, Haverich A. Total aortic arch replacement with a novel four-branched frozen elephant trunk graft: first-in-man results. Eur J Cardiothorac Surg. 2013;43:406-10.

10. Khaladj N, Shrestha M, Peterss S, Strueber M, Karck M, Pichlmaier M, et al Ascending aortic cannulation in acute aortic dissection type A: the Hannover experience. Eur J Cardiothorac Surg. 2008;34:792-6.

11. Yan TD, Bannon PG, Bavaria J, Coselli JS, Elefteriades JA, Griepp RB, et al Consensus on hypothermia in aortic arch surgery. Ann Cardiothorac Surg. 2013;2:163-8.

12. Martens A, Koigeldiyev N, Beckmann E, Fleissner F, Kaufeld T, Krueger H, et al Do not leave the heart arrested. Non-cardioplegic continuous myocardial perfusion during complex aortic arch repair improves cardiac outcome. Eur J Cardiothorac Surg. 2016;49:141-8.

13. Dohle DS, Tsagakis K, Janosi RA, Benedik J, Kuhl H, Penkova L, et al. Aortic remodelling in aortic dissection after frozen elephant trunk. Eur J Cardiothorac Surg. 2016;49:111-7.

14. Grabenwoger M, Alfonso F, Bachet J, Bonser R, Czerny M, Eggebrecht H, et al. Thoracic Endovascular Aortic Repair (TEVAR) for the treatment of aortic diseases: a position statement from the European Association for Cardio-Thoracic Surgery (EACTS) and the European Society of Cardiology (ESC), in 
collaboration with the European Association of Percutaneous Cardiovascular Interventions (EAPCI). Eur J Cardiothorac Surg. 2012;42:17-24.

15. Leontyev S, Misfeld M, Daviewala P, Borger MA, Etz CD, Belaev S, et al. Earlyand medium-term results after aortic arch replacement with frozen elephant trunk techniques-a single center study. Ann Cardiothorac Surg. 2013;2:606-11.

16. Leontyev S, Tsagakis K, Pacini D, Di BR, Mohr FW, Weiss G, et al. Impact of clinical factors and surgical techniques on early outcome of patients treated with frozen elephant trunk technique by using EVITA open stent-graft: results of a multicentre study. Eur J Cardiothorac Surg. 2016;49:660-6.

17. Dohle DS, Tsagakis K, Ibrahim S, Plicht B, Jakob H. Controlled delayed aortic repair in acute aortic syndrome and multiorgan failure: an option in selected cases. Thorac Cardiovasc Surg Rep. 2015;4:52-5.

18. Tsagakis K, Dohle D, Benedik J, Lieder H, Jakob H. Overall Essen's experience with the E-vita open hybrid stent graft system and evolution of the surgical technique. Ann Cardiothorac Surg. 2013;2:612-20.

19. Ius F, Fleissner F, Pichlmaier M, Karck M, Martens A, Haverich A, et al. Total aortic arch replacement with the frozen elephant trunk technique: 10-year follow-up single-centre experience. Eur J Cardiothorac Surg. 2013;44:949-57.

Key Words: aortic arch surgery, frozen elephant trunk, FET, branched graft technique, acute aortic dissection, hybrid prosthesis

\section{Discussion}

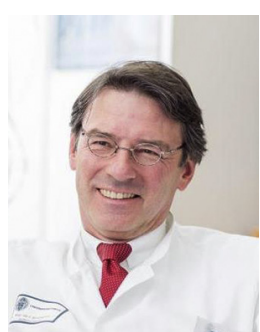

Dr Friedhelm Beyersdorf (Freiburg, Germany). Thank you very much for this presentation. We are also using the Thoraflex device (Vascutek, Inchinnan, Scotland) for frozen elephant trunk implantations, and I agree with all the very good results that you had. We are also very pleased with this relatively new device.

I have 3 questions for you. Because you are using beating heart operations and also cardioplegia, please discuss a little bit what the advantages of the beating heart procedure are in this context.

The second question is, because there is a collar on this device, it is very easy to perform the distal anastomosis, which is usually done distal of the subclavian artery. But one could, of course, also do it between the left carotid artery and the subclavian artery, which might be even easier, and please tell us your experience for this more proximal anastomosis.

And the third question: You mentioned that you are using an endoscope to check the position of the frozen elephant trunk. In what percentage of all your patients in whom you are using endoscopy do you find some abnormal results by the endoscope?

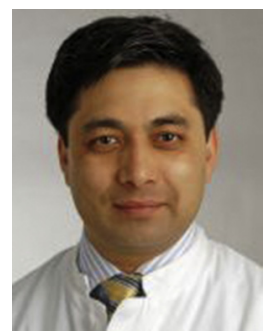

Dr Malakh Lal Shrestha (Hannover, Germany). Thank you. The first question, the beating heart technique, you saw that we had 40 patients with aortic root replacements. So what we initially do is we go on pump, start cooling the patients to $25^{\circ} \mathrm{C}$, and during this time, we do the root. Of course, in this first part, you have to give cardioplegia. But immediately after finishing the root, we start the myocardial perfusion where we control the pressure in the root, we control the flow, and also it is important to totally vent the heart so that the heart is totally empty. So at least at that part, if you want to do the total arch again, you do not have to worry about very long ischemia times, and then you can take your time to do the arch.

This is the big advantage because most of the time if you want to do the David procedure with single arch and double bypasses and then do a total arch, you may end up with a longer crossclamp time, which we do not want. That was the reason for myocardial perfusion.

Now we try always to do the anastomosis before the left subclavian, in fact, between the left carotid and the left subclavian. If it is not possible, we will go beyond that.

Of course we have used the endoscope, but until now we have not had to change anything. It is just a security thing.

Dr Beyersdorf. This was my impression, too, that the frozen elephant trunk fits so very well that malposition is rather rare. But still I think to check it by endoscope is not a bad idea.

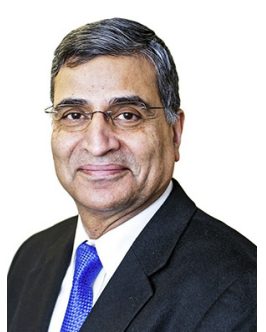

Dr Bansi Koul (Lund, Sweden). A huge experience and a wonderful presentation and very good results. However, we are discussing basically arch replacement under elective circumstances and it is quite a safe operation today.

I noticed that $25 \%$ of your patients experienced recurrent nerve paralysis. I refer again to what Professor Beyersdorf said: You could make arch anastomosis proximally to avoid that complication, and it makes the operation also easier.

Secondly, there is a subset of patients in whom you may perhaps need a prophylactic free elephant trunk for exclusion of future dilatation of the descending aorta by catheter technique. In this subset, you could avoid the possibility of the elephant trunk stimulating the rapid growth of the dilated descending aorta by incorporating a segment of the vascular graft proximal or distal to the left subclavian artery, as you showed. That way you are not committed to the elephant trunk right from the beginning, and you could still have a vascular prosthesis 5 to $6 \mathrm{~cm}$ in length as proximal landing site for a future stent graft in the dilated descending thoracic aorta. Did I make myself understandable?

Dr Shrestha. Yes, but these are the patients we are not doing for prophylaxis care, so most of these patients are either type A dissections or aneurysm of the descending. That is why we are very liberal with the open surgery. If you have to do a total arch replacement, I think, at least our experience has been that we do elephant trunk. 
Dr Koul. Yes. You showed a subset of patients especially with the atherosclerotic aneurysms in whom you had dilatation of the descending aorta distal to the stent graft. My comment refers to that subset.

Dr Shrestha. The maximum size of the graft is $4 \mathrm{~cm}$, and these patients had aortic arch aneurysms, so you had to replace the aortic arch. In these patients because you have to do a total arch replacement, then you put in an elephant trunk.

Dr Koul. Lovely presentation. Thank you.

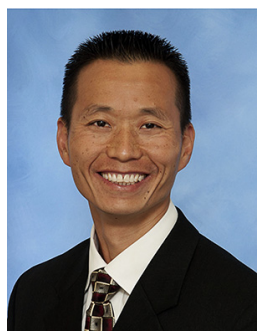

Dr Bo Yang (Ann Arbor, Mich). Do you have a selection of type A dissection patients for the Thoraflex, or do you use it for all type A dissection patients?

Dr Shrestha. We use only for type I dissections, and if the patient is, I would say, below 70. And if someone is 85 , then of course we do not do that. What we try to do is that only surgeons who have done at least 10,15 , or 20 total arch replacements with frozen elephant trunks electively come in.

Both of us are here, so if there is a dissection in Hannover today, there will not be a frozen elephant trunk. Otherwise, one of us always goes in whether we are on call or not.

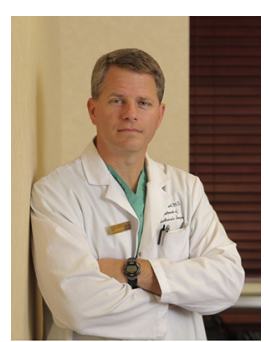

Dr Leonard Girardi (New York, $N Y$ ). One of the things that always concerned me with the frozen elephant trunk as opposed to a standard elephant trunk is this risk of paraplegia and paraparesis.

Essentially this is a prophylactic operation. In $20 \%$ of your patients, you anticipate further interventions relatively soon after the procedure, but the rest of them, $80 \%$ of them, are getting something just in case.

Have you been able to look at patients who have had spinal cord injury, because this keeps coming up in all the articles about frozen elephant trunk. Have you looked at those patients at all to try to predict on whom we should not we do this technique? Worry about the second procedure when the time comes and try to avoid this sort of unacceptable risk of spinal cord injury?

Dr Shrestha. I think it is more a question of how you do it than rather when not to do it. And what we learned now is that the frozen elephant trunk, especially in aneurysms and acute dissections, should not be more than $10 \mathrm{~cm}$ beyond the left subclavian. So we try to make it only $10 \mathrm{~cm}$ at the most. The second is that we always do cerebrospinal fluid drainage.

Third is that is very important because these patients have high blood pressure preoperatively, so immediately after the surgery, you have to keep these patients, I would say, at least under $30 \mathrm{~mm}$ systolic blood pressure, and I would say at least 90 mean pressure.

If you do these things, I think at least in our series now in the past 2 years we have not had paraplegia.

Dr Girardi. So you put in a spinal drain before taking these patients to the operating room?

Dr Shrestha. Yes.

Dr Girardi. In anybody you are anticipating to require a frozen elephant trunk?

Dr Shrestha. No. In every patient in the frozen elephant trunk group. Luckily we are a big university clinic, so even at 2 in the morning when someone comes with type A dissection, there is a surgeon available. It is just luck. 
04/2010 - 10/2014

Total aortic arch + prox. desc. aortic repair $\mathrm{n}=136$

excluded $(n=36)$

- custom made FET/aortic arch graft $(n=17)$

Haverich-Chavan $(n=3)$

Jotec E-Vita + Siena $(n=10)$

Medtronic Valiant + Siena $(n=4)$

- classical ET $(n=19)$

\section{Vascutek Thoraflex Hybrid \\ branched FET graft \\ $n=100$}

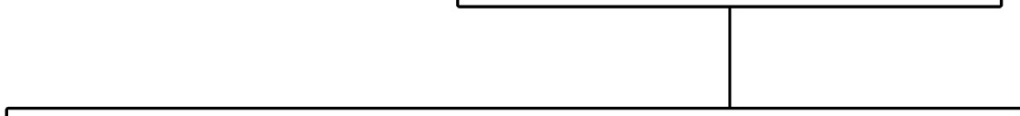

\section{in hospital}

\begin{tabular}{|c|c|c|}
\hline Acute dissection ( $n=37$ ) & Chronic dissection $(n=31)$ & Aneurysm (n=32) \\
\hline pre-op CTA $(n=37 ; 100 \%)$ & pre-op CTA $(n=31 ; 100 \%)$ & pre-OP CTA $(n=32 ; 100 \%)$ \\
\hline $\begin{array}{l}\text { in hospital mort. }(n=3 ; 8 \%) \\
\text { discharged }(n=34,92 \%)\end{array}$ & $\begin{array}{l}\text { in hospital mort. }(n=1 ; 3 \%) \\
\text { discharged }(n=30,97 \%)\end{array}$ & $\begin{array}{l}\text { in hospital mort. }(n=3 ; 9 \%) \\
\text { discharged }(n=29,91 \%)\end{array}$ \\
\hline CTA@ discharge ( $n=32 / 34 ; 94 \%)$ & CTA @ discharge (n=28/30; 93\%) & CTA@ discharge $(n=28 / 29 ; 97 \%)$ \\
\hline
\end{tabular}

\section{Follow Up}

Acute dissection $(\mathbf{n}=34)$
median FU $2.7(2.0-4.7)$ y
FU mortality $(n=3 / 34 ; 9 \%)$
Pat. with FU CTA $(n=28 / 34 ; 82 \%)$
Number of CTAs: $n=64 ; 2.7 /$ Pat.

\section{Chronic dissection $(n=30)$}

median FU $2.7(1.7-4.5)$ y

FU mortality $(n=4 / 30 ; 13 \%)$

Pat. with FU CTA $(n=26 / 30 ; 87 \%)$

Number of CTAs: $n=48 ; 1.8 /$ Pat.

\section{Aneurysm $(n=29)$ \\ median FU 2.5 (1.7-4.6) y \\ FU mortality $(n=5 / 29 ; 17 \%)$ \\ Pat. with FU CTA ( $n=21 / 29 ; 72 \%$ ) Number of CTAs: $n=42 ; 2.0 /$ Pat.}

FIGURE E1. Synopsis showing study cohort and follow-up. FET, Frozen elephant trunk; ET, elephant trunk; CTA, computed tomography angiography; $F U$, follow-up. 